\title{
Gluon Structure Function in Hamiltonian Lattice QCD
}

\author{
H.J. Pirner*, ${ }^{*} b$ D. Grünewald ${ }^{c}$ and E.-M. Ilgenfritz ${ }^{a}$ \\ ${ }^{a}$ Heidelberg University, Institute for Theoretical Physics, 69120 Heidelberg, Germany \\ ${ }^{b}$ Max Planck Institute for Nuclear Physics, 69117 Heidelberg, Germany \\ ${ }^{c}$ Fraunhofer ITWM, 67663 Kaiserslautern, Germany \\ E-mail: piratphys.uni-heidelberg.de, \\ daniel.gruenewald@itwm. fraunhofer.de, \\ ilgenfri@physik.hu-berlin.de
}

We calculate the gluon structure function of a color dipole in a new approach evaluating the matrix elements of $S U(2)$ gluon field operators separated along a direction close to the light cone. As vacuum state in the pure glue sector, we use a variational ground state of the near-light-cone Hamiltonian. With a mean momentum fraction of the gluons fixed to the "experimental value" in a proton, the resulting gluon structure function for a dipole state with four links is compared qualitatively to the NLO MRST 2002 parameterization at $Q^{2}=1.5 \mathrm{GeV}^{2}$.

PACS : $11.15 . \mathrm{Ha}, 02.70 . \mathrm{Ss}, 11.80 . \mathrm{FV}$

Light Cone 2010 - LC2010

June 14-18, 2010

Valencia, Spain

${ }^{*}$ Speaker. 
In Euclidean lattice simulations, using the operator product expansion the lowest moments of the meson and nucleon structure functions have been evaluated [1, 2, 3, 4]. On the other side, Loop-loop correlation functions of tilted Wegner-Wilson loops computed in Euclidean space [6, 5] can be related to the gluon distribution function of a color dipole which consists of a static quark and antiquark pair connected by a Schwinger string. Alternatively, an approach based on light cone dynamics $[8,7]$ refers to constituents moving along the light cone, as suggested by the picture of Feynman scaling (as zeroth approximation). In the light cone approach the non-perturbative QCD vacuum structure is hard to achieve within the Fock representation of free fields acting on a trivial vacuum. As we will demonstrate, the confining nontrivial QCD vacuum is essential to generate the correct interaction of colored constituents moving along light like trajectories.

Therefore we have developed a near-light-cone (nlc) approach in which we exploit the lattice formulation given for $S U(2)$ gluodynamics, benefitting from simplifications emerging in the light cone limit. In Ref. [9] we have constructed a ground state wave functional of the nlc Hamiltonian which is simpler than the ground state in equal-time theory. In Ref. [10] we have outlined the formalism to determine the gluon distribution function of a color dipole with this ground state wave functional. The present letter gives the main new results which one obtains following this approach.

The gluon structure function $g\left(x_{B}\right)$ is the probability that a gluon carries a fraction $x_{B}$ of the longitudinal momentum of the fast moving hadronic target. In light cone coordinates, it is given by the Fourier transform of the matrix element of the two-point operator $G_{l c}\left(z^{-}, \vec{z}_{\perp} ; 0, \vec{z}_{\perp}\right)$ of longitudinally separated gluon field strength operators in a hadron state $\left|h\left(p_{-}, \overrightarrow{0}_{\perp}\right)\right\rangle$ :

$$
g\left(x_{B}\right)=\frac{1}{x_{B}} \frac{1}{2 \pi} \int_{-\infty}^{\infty} d z^{-} d^{2} \vec{z}_{\perp} e^{-\mathrm{i} x_{B} p_{-} z^{-}} \frac{1}{p_{-}}\left\langle h\left(p_{-}, \overrightarrow{0}_{\perp}\right)\left|G_{l c}\left(z^{-}, \vec{z}_{\perp} ; 0, \vec{z}_{\perp}\right)\right| h\left(p_{-}, \overrightarrow{0}_{\perp}\right)\right\rangle_{c},
$$

with

$$
G_{l c}\left(z^{-}, \vec{z}_{\perp} ; 0, \vec{z}_{\perp}\right)=\sum_{k=1}^{2} F_{-k}^{a}\left(z^{-}, \vec{z}_{\perp}\right) S_{a b}^{A}\left(z^{-}, \vec{z}_{\perp} ; 0, \vec{z}_{\perp}\right) F_{-k}^{b}\left(0, \vec{z}_{\perp}\right) .
$$

The hadron $\left|h\left(p_{-}, \overrightarrow{0}_{\perp}\right)\right\rangle$ is centered in transversal configuration space at $\vec{b}_{\perp}=\overrightarrow{0}_{\perp}$ and carries a longitudinal momentum $p_{-}$. The index "c" indicates that the connected matrix element has to be taken. The Schwinger line $S_{a b}^{A}\left(z^{-}, \vec{z}_{\perp} ; 0, \vec{z}_{\perp}\right)$ in the adjoint representation and running along a light like path is inserted between the gluon field strength operators $F_{-k}^{b}\left(0, \vec{z}_{\perp}\right)$ and $F_{-k}^{a}\left(z^{-}, \vec{z}_{\perp}\right)$. The importance of the Schwinger lines along the light cone has been demonstrated e.g. in the looploop correlation model where hadron-hadron scattering cross-sections are calculated from WegnerWilson loop correlation functions [6]. In another language, the eikonal phases arising from the strings along the $x^{-}$- direction describe "final state" interaction effects which distinguish structure functions from parton probabilities [11].

We are using near-light-cone coordinates which allow us to implement light front quantization as a limit of equal time quantization. The definition of the temporal nlc coordinate $x^{+}$contains an additional external parameter $\eta$ which facilitates a smooth interpolation between equal time quantization $\left(\eta=1, x^{+}=x^{0}\right)$ and light cone quantization $\left(\eta=0, x^{+}=1 / 2\left(x^{0}+x^{3}\right)\right)$. The definitions are:

$$
x^{+}=\frac{1}{2}\left[\left(1+\eta^{2}\right) x^{0}+\left(1-\eta^{2}\right) x^{3}\right], x^{-}=\left[x^{0}-x^{3}\right] .
$$


The $\eta \rightarrow 0$ limit can be interpreted as the infinite momentum frame limit in which the partons of the color dipole move with infinite momentum. The use of nlc coordinates has the advantage that no quantum constraint equations have to be solved.

In the $S U(2)$ lattice formulation we have two transversal gauge fields $A_{j}(j=1,2)$ and one longitudinal one $A_{-}(j=-)$ that are represented by the corresponding link matrices $U_{j}(\vec{x})$. The $A_{+}$ component of the gauge field is set equal to zero. As a result one has the Gauss-law constraining the entire Hilbert space to the physical sector of gauge invariant states. The gluon dynamics is determined by the Hamiltonian which has been derived in Ref. [9]. It represents the gluon energy density on the lattice. The QCD coupling constant enters as $\lambda=4 / g^{4}$ in the $S U(2)$ case,

$$
\begin{aligned}
\mathscr{H}_{\text {eff,lat }}= & \frac{1}{N_{-} N_{\perp}^{2}} \frac{1}{a_{\perp}^{4}} \frac{2}{\sqrt{\lambda}} \sum_{\vec{x}}\left\{\frac{1}{2} \sum_{a} \Pi_{-}^{a}(\vec{x})^{2}+\frac{1}{2} \lambda \operatorname{Tr}\left[\mathbb{1}-U_{12}(\vec{x})\right]\right. \\
& \left.+\sum_{k, a} \frac{1}{2} \frac{1}{\tilde{\eta}^{2}}\left[\Pi_{k}^{a}(\vec{x})^{2}+\lambda\left(\operatorname{Tr}\left[\frac{\sigma_{a}}{4 i}\left(U_{-k}(\vec{x})-U_{-k}^{\dagger}(\vec{x})\right)\right]\right)^{2}\right]\right\} .
\end{aligned}
$$

Here, $U_{12}$ are purely transversal plaquettes, and $U_{-k}$ are longitudinal-transversal plaquettes.

The Hamiltonian contains the chromo-electric field strength operators $\Pi_{i}^{a}(\vec{x})$, which are canonically conjugate to the links $U_{i}(\vec{x})$. They obey commutation relations which follow from the corresponding continuum relations,

$$
\left[\Pi_{i}^{a}(\vec{x}), U_{j}(\vec{y})\right]=\left(\sigma^{a} / 2\right) U_{i}(\vec{x}) \delta_{\vec{x}, \vec{y}} \delta_{i, j} .
$$

The constant $\tilde{\eta}$ in Eq. (3) is the product $\tilde{\eta}=\eta \cdot \xi$ of the near-light-cone parameter $\eta$ and an eventual anisotropy parameter $\xi=a_{-} / a_{\perp}$, the ratio of lattice spacings in longitudinal and transverse directions. If one chooses $\eta=1$ and varies $\xi$, one simulates an anisotropic equal time theory. In the limit $\xi \rightarrow 0$ one ends up with a system, which is contracted in the longitudinal direction. Verlinde and Verlinde [12] and Arefeva [13] have advocated such a lattice to describe high energy scattering. A longitudinally contracted system means that even the minimal momenta become high in longitudinal direction. The limit $\xi \rightarrow 0$ leads to the same physics as the light cone limit $\eta \rightarrow 0$ with isotropic lattice spacings in longitudinal and transverse directions. In both cases the nlc Hamiltonian is dominated by the terms proportional to $1 / \tilde{\eta}^{2}$ involving transverse chromo-electric and chromo-magnetic fields. For a nlc-Hamiltonian formulation, the two-point operator in Eq. (2) has to be replaced by

$$
G_{l c}\left(z^{-}, \vec{z}_{\perp} ; 0, \vec{z}_{\perp}\right)=\frac{1}{2} \sum_{k=1}^{2}\left(F_{-k}^{a}\left(z^{-}, \vec{z}_{\perp}\right) S_{a b}^{A}\left(z^{-}, \vec{z}_{\perp} ; 0, \vec{z}_{\perp}\right) \Pi_{k}^{b}\left(0, \vec{z}_{\perp}\right)+\left\{F_{-k}^{a} \leftrightarrow \Pi_{k}^{a}\right\}\right) .
$$

In Ref. [9] we have determined a variational gluonic ground state wave functional $\left|\Psi_{0}\right\rangle$ which consists of a product of single-plaquette wave functionals with two variationally optimized parameters $\rho_{0}=\rho_{0}(\lambda, \tilde{\eta})$ and $\delta_{0}=\delta_{0}(\lambda, \tilde{\eta})$,

$$
\begin{gathered}
\left|\Psi_{0}\right\rangle=\Psi_{0}[U]|0\rangle=\sqrt{N_{\Psi}} e^{f[U]}|0\rangle, \\
f[U]=\sum_{\vec{x}}\left\{\sum_{k=1}^{2} \rho_{0} \operatorname{Tr}\left[U_{-k}(\vec{x})\right]+\delta_{0} \operatorname{Tr}\left[U_{12}(\vec{x})\right]\right\} .
\end{gathered}
$$




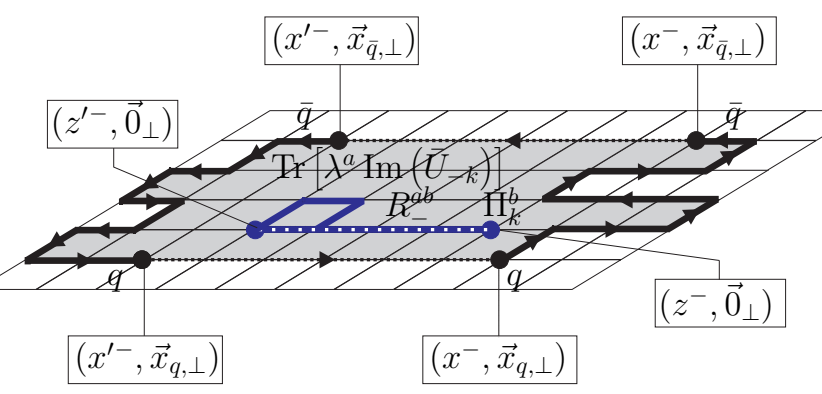

Figure 1: The generalized Wegner-Wilson loop generating the matrix element between the color dipole states. Quark $q$ and antiquark $\bar{q}$ are connected by $n$ transversal links. Only one transversal direction is shown. The insertion represents the gluonic two-point operator with an electric and a magnetic field operator.

$N_{\Psi}$ is a normalization factor. Here, the state $|0\rangle$ represents the trivial ground state which is annihilated by the field momenta $\Pi_{k}^{a}(\vec{x})$ conjugate to the links,

$$
\Pi_{k}^{a}(\vec{x})|0\rangle=0 \text { and }\langle 0| \Pi_{k}^{a}(\vec{x})=0 \text { for } \forall \vec{x}, k, a .
$$

We have optimized this ansatz and extrapolated the parameters $\rho_{0}, \delta_{0}$ to the light cone $\tilde{\eta} \rightarrow 0$ (see Ref. [9]).

Since the nlc Hamiltonian in Eq. (3) contains only gluon fields, we cannot derive a full hadronic wave function from this Hamiltonian. We have to make a model taking care of the gluon structure alone while treating the quarks schematically. Our model represents a dipole localized in transversal configuration space at a fixed center of mass position $\vec{b}_{\perp}=\overrightarrow{0}$. The distance $\vec{d}_{\perp}$ between quark and antiquark is bridged by a Schwinger line along some path $\mathscr{C}_{\perp}$ in the transversal plane. The in and out dipole states form a Wegner-Wilson loop in the eikonal approximation as sketched in Fig. 1.

The longitudinal lattice momenta must be $n$-fold (integer) multiples of $2 \pi / N_{-}$, with $0 \leq n \leq$ $N_{-} / 2-1$, since the longitudinal light cone momentum for an on shell particle is always positive. The momentum $p_{-}$of the target is chosen (in lattice units) as the largest momentum in order to allow for the maximum resolution in the gluon distribution function [14]. The longitudinal lattice gluon momenta then have the resolution

$$
p_{-}=\frac{2 \pi}{N_{-}}\left(\frac{N_{-}}{2}-1\right), \frac{\Delta p_{-}^{g}}{p_{-}}=\frac{2}{N_{-}-2} .
$$

In order to have a high resolution, the extension of the lattice in the longitudinal direction has to be very large.

We impose the quark dynamics of the color dipole externally. Since the total hadron longitudinal momentum is given by the sum of the momenta of its constituents (quarks+gluons), the typical mean gluon momentum is taken from experiment. For a rough qualitative comparison, we use the $M R S T$-parameterization [15] of the proton $S U(3)$ gluon distribution function at the input scale $Q^{2} \approx \pi^{2} / a_{\perp}^{2}=1.5 \mathrm{GeV}^{2}$ corresponding to $\lambda \approx 10$, and assign a mean momentum fraction $p_{-}^{S}=0.38 p_{-}$to the gluon system of the $S U(2)$ color dipole.

The gluon distribution function for a one-link dipole $p_{-}^{g} g_{1}\left(p_{-}^{g} ; p_{-}^{S}\right)$ with total gluonic momentum $p_{-}^{S}$ has been computed on lattices with $N_{-}=20,30,50$, and 100 sites in the longitudinal 


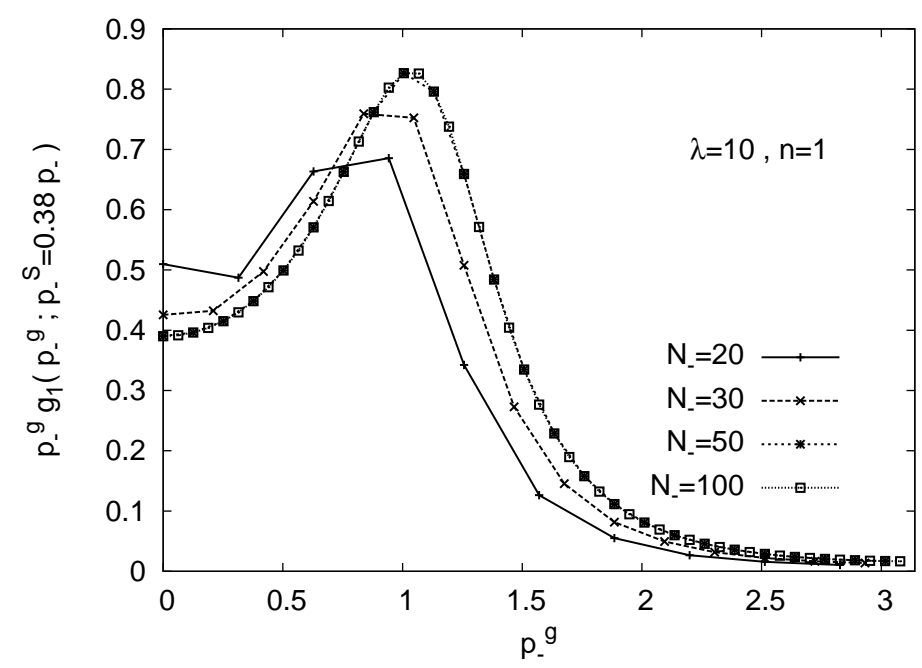

Figure 2: Gluon distribution function $p_{-}^{g} g_{1}\left(p_{-}^{g} ; p_{-}^{S}=0.38 p_{-}\right)$for a single transversal link, $n=1$. The dependence on the longitudinal lattice size $N_{-}$is shown for a lattice coupling $\lambda=4 / g^{4}=10$ (see Eq.(4)). The average gluon momentum $\left\langle p_{-}^{g}\right\rangle=p_{-}^{S}$ was input and adjusted to $\left\langle x_{B}\right\rangle p_{-}=0.38 p_{-}$(see text).

direction. Fig. 2 demonstrates the effect of increasing the number of longitudinal lattice sites, i.e. approaching the infinite volume limit. Scaling for $\lambda=10$ seems to be obeyed for longitudinal lattice extensions larger than $N_{-}=50$. Realistic lattice simulations need quite large longitudinal lattice sizes. The smearing of the distribution function is due to the gluon dynamics incorporated in the Wegner-Wilson loop expectation value. Thus, the area law behavior of the Wegner-Wilson loop yields a non-trivial gluon wave function which broadens the distribution.

For larger $\lambda$, i.e. smaller QCD gauge coupling $g^{2}$, the peak in the one-link distribution function becomes narrower. In the extreme weak coupling limit, when the link reduces to a single gluon, the gluon distribution function becomes totally sharp. On the other hand, for strong coupling one has a broad momentum distribution peaked around $p_{-}^{S}$.

The one-link dipole gluon distribution is the basic building block from which the multiple link dipole gluon distribution function of a hadron can be constructed. The actual hadronic state arises from a superposition of multiple link configurations. Let the wiggly strings $S_{q \bar{q}}$ (c.f. Fig. 1) connecting the quark and antiquark have a fixed number of transversal links since all the dipole configurations with fixed transversal length $n$ have the same energy. Then one rotates the hadron in the transversal plane by summing over randomly chosen curves $\mathscr{C}_{\perp}$, in order to project this state on angular momentum $J_{z}=0$. From the random walk follows that for $n$-links, the hadron has an average radius squared $\vec{R}_{\perp}^{2}$ proportional to $n$ : Hence, the area of the hadron scales with the number of links:

$$
\left\langle R_{\perp}^{2}\right\rangle=n a_{\perp}^{2} / 2
$$

Due to small $\delta_{0}$ in the light cone limit, the ground state wave functional allows for ordinary strong coupling methods. This implies especially that one needs incoming and outgoing states sharing the same transversal links connecting the quark and antiquark. Therefore, the gluon distribution can be obtained for a special string elongated along only one of the transversal axes (c.f. Fig. 1).

The computation of the $\mathrm{n}$-link gluon distribution function $g_{n}$ is done in analogy to the compu- 


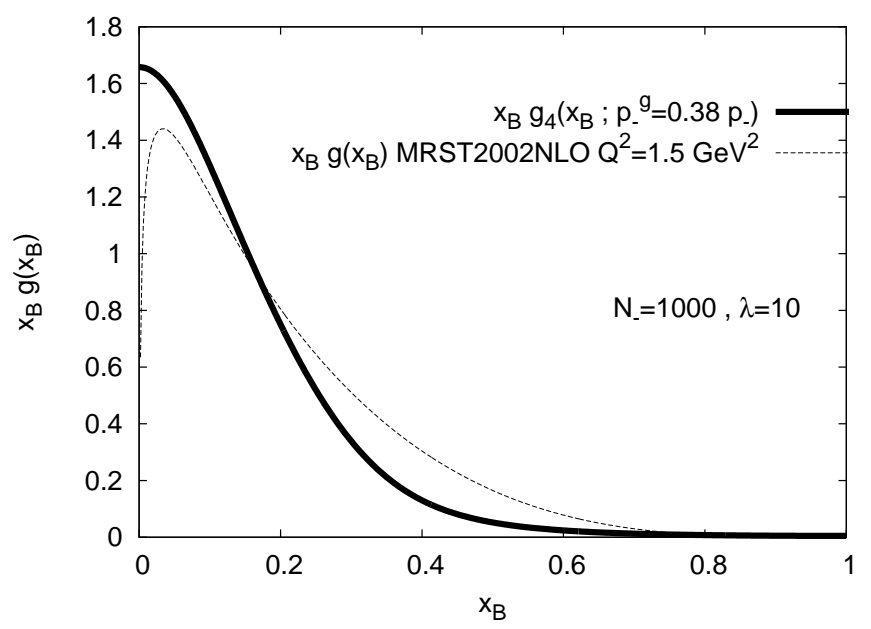

Figure 3: Qualitative comparison of the gluon distribution function $x_{B} g_{n}\left(x_{B} ; p_{-}^{g}\right)$ for $n=4$ transversal $S U$ (2) links with the MRST gluon distribution function of a proton at $Q^{2}=1.5 \mathrm{GeV}^{2}$. The average gluon momentum was taken as input for the lattice calculation on a $N_{-}=1000$ lattice at gauge coupling $\lambda=10$.

tation of the single link gluon distribution. In strong coupling the total loop factorizes, therefore the $n$-link distribution function is given by the product of a splitting function $P_{n \rightarrow n-1}$ multiplying the gluon distribution function with $n-1$ links. In the emerging recursion relation all possible intermediate momenta of the substring are summed over:

$$
g_{n}\left(p_{-}^{g} ; p_{-}^{S}\right)=\frac{2 \pi}{N_{-}} \sum_{p_{-}^{S^{\prime}=0}}^{p_{-}^{S}} g_{n-1}\left(p_{-}^{g} ; p_{-}^{S \prime}\right) P_{n \rightarrow n-1}\left(p_{-}^{S}, p_{-}^{S \prime}\right)
$$

The splitting function $P_{n \rightarrow n-1}\left(p_{-}^{S}, p_{-}^{S \prime}\right)$ denotes the probability that a string with $n$ transversal links and total momentum $p_{-}^{S}$ splits into a string with $n-1$ transversal links and total momentum $p_{-}^{S \prime}$ and is given in Ref. [10]. The initial condition for the recursion relation Eq. (9) is given by the onelink dipole function $g_{1}\left(p_{-}^{g} ; p_{-}^{S}\right)$. The computation is purely arithmetic for small $\delta_{0}$, and we can use a large longitudinal lattice with $N_{-}=1000$ lattice sites. If one increases the number of transversal links, the gluons have access to a larger region in phase space due to the splitting function $P_{n \rightarrow n-1}$ in Eq. (9). Therefore the total gluon momentum will be partitioned among more gluons. Hence, it becomes more likely to find a gluon with a small fraction of the total momentum.

In Fig. 3, we show the theoretical gluon structure function for a $n=4 S U(2)$ link dipole as a function of the gluon fractional momentum $x_{B}=p_{-}^{g} / p_{-}$. To have a rough qualitative comparison, we also show the MRST gluon distribution function at $Q^{2}=1.5 \mathrm{GeV}^{2}$ which is for real protons and $S U(3)$. As before, the first moment of the lattice gluon distribution function has been fixed in this figure to the value $\left\langle x_{B}\right\rangle=0.38$ at $Q^{2}=1.5 \mathrm{GeV}^{2}$. We choose four links to be consistent with the size of a proton and the relation $\left\langle R_{\perp}^{2}\right\rangle=n a_{\perp}^{2} / 2$ and a transversal lattice spacing of $a_{\perp} \approx$ $0.65 \mathrm{fm}$. The lattice gluon distribution function for a color dipole shows a similar behavior as the phenomenological MRST-gluon distribution function for a proton.

The model presented here also shows that $x_{B} g\left(x_{B}\right)$ for the gluon at small $x_{B}$ becomes proportional to the hadronic size $R_{\perp}^{2}$. This coincides with the empirical soft Pomeron behavior of hadronic 
cross sections. To evolve the structure function with increasing resolution $Q^{2}$ and with decreasing $x_{B}$ in Hamiltonian lattice QCD, one needs a more sophisticated ground state wave functional respecting scaling with the lattice spacing.

\section{References}

[1] D. Dolgov et al. [LHPC collaboration and TXL Collaboration], Moments of nucleon light cone quark distributions calculated in full lattice QCD, Phys. Rev. D 66 (2002) 034506 [ hep-lat/ 0201021 ].

[2] M. Göckeler, R. Horsley, D. Pleiter, P. E. L. Rakow and G. Schierholz [QCDSF Collaboration], A lattice determination of moments of unpolarised nucleon structure functions using improved Wilson fermions, Phys. Rev. D 71 (2005) 114511 [hep-ph/ 0410187$].$

[3] M. Göckeler et al. [QCDSF Collaboration], A preliminary lattice study of the glue in the nucleon, Nucl. Phys. Proc. Suppl. 53 (1997) 324 [hep-lat/9608017].

[4] H. B. Meyer, J. W. Negele, Gluon contributions to the pion mass and light cone momentum fraction, Phys. Rev. D 77 (2008) 037501 [0707.3225 [hep-lat] ].

[5] M. Giordano, E. Meggiolaro, High-energy hadron-hadron (dipole-dipole) scattering from lattice QCD, [0808.1022 [hep-lat]].

[6] A. I. Shoshi, F. D. Steffen, H. G. Dosch and H. J. Pirner, Confining QCD strings, Casimir scaling, and a Euclidean approach to high-energy scattering, Phys. Rev. D 68 (2003) 074004 [hep-ph/0211287].

[7] S. Dalley, Light cone physics: Hadrons and beyond: Proceedings, (2003).

[8] H. W. L. Naus, H. J. Pirner, T. J. Fields and J. P. Vary, QCD near the light cone, Phys. Rev. D 56 (1997) 8062 [hep-th/9704135].

[9] D. Grünewald, E.-M. Ilgenfritz, E. V. Prokhvatilov and H. J. Pirner, Formulating Light Cone QCD on the Lattice, Phys. Rev. D 77 (2008) 014512 [ 0711.0620 [hep-lat] ].

[10] D. Grünewald, E.-M Ilgenfritz and H. J. Pirner, Gluon Structure Function of a Color Dipole in the Light-Cone Limit of Lattice QCD, [0908.0715 [hep-th] ].

[11] S. J. Brodsky, P. Hoyer, N. Marchals, S. Peigne and F. Sannino, Structure functions are not parton probabilities, Phys. Rev. D 65 (2002) 114025 [hep-ph / 0104291 ].

[12] H. Verlinde, E. Verlinde, $Q C D$ at high-energies and two-dimensional field theory, [hep-th/9302104].

[13] I. Y. Arefeva, Large N QCD at high-energies as two-dimensional field theory, Phys. Lett. B 328 (1994) 411 [hep-th/9306014].

[14] M. Burkardt, S. Dalley, The relativistic bound state problem in QCD: Transverse lattice methods, Prog. Part. Nucl. Phys. 48 (2002) 317 [hep-ph / 0112007 ].

[15] A. D. Martin, R. G. Roberts, W. J. Stirling and R. S. Thorne, Uncertainties of predictions from parton distributions. 1: Experimental errors, Eur. Phys. J. C 28 (2003) 455 [hep-ph/ 0211080 ]. 